\title{
Effect of Irradiation Damage on the Shear Strength of Cu-Nb Interfaces
}

\author{
Shimin Mao ${ }^{1}$, Sezer Ozerinc ${ }^{2}$, William P. King ${ }^{2}$, Robert S. Averback ${ }^{1,3}$, Shen J.
} Dillon $^{1,3}$

\author{
1. Department of Materials Science and Engineering, University of Illinois Urbana \\ Champaign, 61801, IL, USA \\ 2. Department of Materials Science and Engineering, University of Illinois \\ Urbana Champaign, 61801, IL, USA
3. Frederick Seitz Materials Research Laboratory, University of Illinois Urbana Champaign, 61801, IL, USA

\begin{abstract}
The influence of irradiation induced damage on interfacial shear strength of $\mathrm{Cu}-\mathrm{Nb}$ interfaces was characterized via compression of nanolaminate pillars performed in-situ in a transmission electron microscope. Chemical mixing and interfacial roughening during $\mathrm{MeV} \mathrm{Kr}$ ion irradiation leads to increased interfacial shear strength by as much as $60 \%$, from 0.6 GPa for the as-deposited material to $0.95 \mathrm{GPa}$ for samples irradiated at liquid nitrogen temperature. The increase in interfacial shear strength was most pronounced at low temperatures $\left(\approx-196^{\circ} \mathrm{C}\right)$, but it is still significant at $\approx 300^{\circ} \mathrm{C}$. This observation was correlated with increased chemical mixing at lower temperatures, as determined from compositional profiles characterized by energy dispersive spectroscopy.
\end{abstract}

Efforts to engineer alloys tolerant to prolonged exposure to energetic particle irradiation have focused in recent years on creating high densities of interfaces to provide neutral sinks for point defect annihilation. Nanostructured materials afford a natural route to achieve this goal, with multiphase nanostructured materials such as ODS alloys[1], nanolaminates [2], and self-organizing immiscible alloys[3, 4] currently appearing most promising. How well the microstructural and mechanical stability of these materials will persist in harsh thermal, chemical, and irradiative environments envisioned in advanced nuclear reactor, however, remains uncertain [5] . One crucial step toward understanding the reliability of these materials will be elucidating the deformation response of hetero-interfaces, as these interfaces are likely to control the overall mechanical properties and phase stability of these materials. Indeed numerous computational and experimental studies have begun to explore the properties of these hetero-interfaces.[6-8]

Nanostructured alloys typically obey a Hall-Petch-like behavior until their microstructural features fall below some critical wavelength, at which point their strength tends to saturate, or even decrease.[9] This has been investigated in detail in nanolaminates grown by physical vapor deposition, as the interfaces of these structures can be controlled precisely. Work on these systems suggests that the ultimate limitation bounding increased Hall-Petch strengthening is determined by the nature of slip across the interface and shearing in the interfacial plane.[9] Pristine interfaces have received the 
most attention in this regard,[10] as they are most conducive to atomistic simulations. Damage accumulation during irradiation, however, is anticipated to impact dislocation motion in and across individual interfaces. This is because planar immiscible heterophase interfaces tend to chemically mix and/or roughen under irradiation[11, 12], and molecular dynamics (MD) simulations indicate that the interfacial shear strength of model $\{111\}$ Kurdumov-Sachs (K-S) oriented fcc-bcc interfaces can vary by as much as 60-160\%, depending on shear direction, as a result of chemical mixing.[6] It is also anticipated that interfacial roughening will impact interfacial sliding and dislocation slip across the interface, although the effects of roughening are more difficult to treat by MD, owing to the long wavelengths involved.

Past work has demonstrated that immiscible alloys such as $\mathrm{Cu}-\mathrm{Nb}$ tend to mix chemically during irradiation at low temperatures due to ion beam mixing. $[11,12]$ At higher temperatures, room temperature and above, mixing is reduced by radiationenhanced diffusion which leads to segregation in this highly immiscible alloy.[11] Some roughening of the interface may also take place during irradiation, but this has not been carefully investigated. The present work investigates how damage accumulation at immiscible fcc-bcc interfaces during irradiation at different temperatures affects interfacial sliding under shear using in-situ TEM-based nanocompression experiments. The technique directly correlates critical events in the stress-strain curve to the associated plasticity in the microstructure, thereby enabling the geometric factors governing the resolved shear stress to be measured accurately. $\mathrm{Cu}-\mathrm{Nb}$ was selected as a model fcc-bcc interface owing to the vast amount of information available on the structure and strength of these interfaces in absence of irradiation; preliminary measurements on $\mathrm{Cu}-\mathrm{V}$ and $\mathrm{Ni}$ $\mathrm{Nb}$ are also briefly discussed.

To promote interfacial shearing, samples were prepared with their interfaces inclined at either $30^{\circ}$ or $45^{\circ}$ to the loading plane, as shown in supplementary Fig. S1. A focused ion beam (FIB: FEI 600i company) was used to prepare $\approx 5 \mu \mathrm{m}$ pre-tilted pillars from commercial wedge-shaped Si substrates (Hysitron). Multilayers of Cu (99.99\% Lesker) and $\mathrm{Nb}(99.95 \%$ Lesker) were subsequently grown on these pillars using magnetron sputtering in $2 \times 10^{-3}$ torr Ar in a chamber with a base pressure of $\approx 10^{-8}$ torr. Preliminary tests were also performed on $\mathrm{Cu}-\mathrm{V}(99.5 \%)$ and $\mathrm{Ni}(99.99 \%)-\mathrm{Nb}$ grown under similar conditions. Electron diffraction indicated that the layers generally grew with the K-S orientation relationship: $\mathrm{Cu}(111) / / \mathrm{Nb}$ (110), which has been observed previously.[13] Samples comprised of alternating $50 \mathrm{~nm} \mathrm{Cu}$ and $50 \mathrm{~nm} \mathrm{Nb}$ layers were grown to a total thickness of 2-3 $\mu \mathrm{m}$. The average grain size within each layer was $\sim 100 \mathrm{~nm}$. After thinfilm deposition, the FIB was utilized to thin the samples to their final diameter, $\approx 250 \mathrm{~nm}$. The nanopillars were then irradiated with $1.8 \mathrm{MeV} \mathrm{Kr}^{+}$using a current density of $1.4 \mathrm{~mA}$ $\mathrm{cm}^{-2}\left(2 \times 10^{15}\right.$ ions $\left./ \mathrm{cm}^{2}\right)$ at $-196,27$, and $300^{\circ} \mathrm{C}$ to doses between 5 and $50 \mathrm{dpa}$ (displacements per atom). The irradiation flux was perpendicular to the pillar axis. The irradiation provided reasonably uniform damage throughout the sample $(+/-20 \%$ as calculated by SRIM [14], but see also Ref. [15] for more details relevant to our test geometry). After irradiation, the FIB was used again to reduce the total height of the samples, such that the aspect ratio was $\approx 4$. This cutting procedure also provided a smooth planar surface for contacting the flat diamond punch (see below). High-angle 
annular dark field scanning transmission electron microscopy (HAADF-STEM) and energy dispersive spectroscopy were performed in a $200 \mathrm{kV}$ JEOL 2010FS.

The in-situ nanocompression experiments were carried out in a $200 \mathrm{kV}$ JEOL $2010 \mathrm{LaB}_{6}$ TEM using a Hysitron PI-95 picoindenter. The calibration of the picoindenter was tested by compressing identical $5 \mu \mathrm{m}$ Si beams in situ using the PI-95 and ex situ using a Hysitron TI-950 triboindentor, which was calibrated against standard quartz. The moduli in the two tests agree to within $10 \%$ (see Fig. S2). Samples were compressed in situ in the displacement control mode at a rate of $1 \mathrm{~nm} \mathrm{~s}^{-1}$ using a $3 \mu \mathrm{m}$ square flat diamond punch (Micro Star Tech, Inc). At least 3 samples were tested for each condition; we report here the mean values and standard deviations as error.

Figure 1 (also Video $\mathrm{S} 1$ ) provides a representative example of the nanocompression test and the associated load-displacement curve. The onset of yielding in the loaddisplacement curve corresponds to the initiation of interfacial sliding at the first $\mathrm{Cu}-\mathrm{Nb}$ interface near the tip of the sample. After the first $\mathrm{Nb}$ layer slides away, a second interfacial sliding event occurs at the second $\mathrm{Cu}-\mathrm{Nb}$ interfaces, which corresponds to the reduction of load in the load-displacement curve. Due to the tapered geometry of the samples, interfacial layers typically shear first in the thinnest region near the end of the tip. The cross sections of the samples are approximately circular at any distance from the end of the pillar, and the cross-sectional area in the region where shear occurs was measured just prior to shear initiation in order to calculate the resolved shear stress:

$$
\mathrm{G}=\frac{\mathrm{F} \cos \alpha \cos \Phi}{\pi \mathrm{R}^{2}}
$$

where $\mathrm{F}$ is the force, $\mathrm{R}$ is the radius of the sample, and $\cos \alpha \cdot \cos \Phi$ is the $\mathrm{Schmid}$ factor. At least three measurements were performed for each sample and an average value of $\Phi$, the projected shear angle, of $60^{\circ}$ was used to calculate the shear strength. Measurements on several different samples produced variations $<10 \%$, suggesting that approximating the in-plane component as an average isotropic value is reasonable. This approximation is further supported by noting that the sample diameter exceeds the grain size by at least a factor of two, leading to an averaging effect over multiple slip directions. As-deposited $\mathrm{Cu}-\mathrm{Nb}$ samples approximately 250, 500, and $1000 \mathrm{~nm}$ in diameter were tested with inclination angles of both $30^{\circ}$ and $45^{\circ}$. The samples all exhibited similar interfacial shear strengths $0.60 \pm 0.05 \mathrm{GPa}$ (e.g. see Fig. S3), where the error is reported as one standard deviation. These results indicate that size effects are insignificant on these length scales. Our value for the interfacial shear strength agrees well with the value of $0.55 \mathrm{GPa}$ measured ex situ by $\mathrm{Li}$ et al. [10]. For $\mathrm{Cu}-\mathrm{V}$ and $\mathrm{Ni}-\mathrm{Nb}$, shear did not occur at the interface, but rather in bulk sample, (see Fig. S4-S5), and so these systems will not be considered here further.

Representative examples of in-situ tests performed on irradiated samples are provided in the supplemental data (Fig. S6-S11). Figure 2 summarizes the measured Cu$\mathrm{Nb}$ interfacial shear strengths associated with samples irradiated to different doses at liquid nitrogen temperature, room temperature, and $300^{\circ} \mathrm{C}$. At $300^{\circ} \mathrm{C}$, the interfacial shear strength increases to $0.70 \pm 0.03 \mathrm{GPa}$ after a dose of $5 \mathrm{dpa}$ and then remains constant $(0.68 \pm 0.09 \mathrm{GPa})$ on increasing the dose to $50 \mathrm{dpa}$. Irradiation to $5 \mathrm{dpa}$ at room 
temperature leads to an increase in shear strength to $0.85 \pm 0.10 \mathrm{GPa}$ and marginally higher, $0.92 \pm 0.03 \mathrm{GPa}$, after $50 \mathrm{dpa}$. Finally, after irradiation at liquid nitrogen temperature, the interfacial shear strength increases to $0.95 \pm 0.11 \mathrm{GPa}$ at $5 \mathrm{dpa}$ and $0.94 \pm 0.08 \mathrm{GPa}$ at $10 \mathrm{dpa}$. The shear strength thus tends to increase with decreasing irradiation temperature, saturating at each temperature by a dose of $\approx 5 \mathrm{dpa}$. The maximum increase in interfacial shear strength due to damage accumulation via mixing and/or roughening is thus approximately $60 \%$.

These experimental results can be compared with past MD simulations.[6] These simulations have predicted that the interface shear strengths of perfectly flat $\mathrm{Cu}-\mathrm{Nb} \mathrm{K}-\mathrm{S}$ interfaces along the [11-2] and [1-10] directions are approximately 0.6 and $0.3 \mathrm{GPa}$ respectively [6]. The interface in this study was then made diffuse by creating an interfacial layer composed of 3 atomic layers on each side of the central of plane with each atomic layer having compositions $\mathrm{X}, \mathrm{X} / 2$, and $\mathrm{X} / 4$, where $\mathrm{X}$ is the molar fraction of $\mathrm{Nb}$ or $\mathrm{Cu}$. For $\mathrm{X}=0.25$ the shear strength in these simulations increased to 0.95 and 0.8 $\mathrm{GPa}$, respectively. These calculated values of the shear strength of diffuse interfaces thus agree quite well with our experiments, which we anticipate to, also exhibit diffuse chemical mixing resulting from irradiation. Examination of the interface structure in these simulations, furthermore, showed that for $\mathrm{X}=0.25$ the interface was amorphous.

In order to further validate the correlation between the MD simulations and experiments, the local chemistry and morphology of the irradiated interfaces were characterized. HAADF-STEM images of the irradiated samples reveal that the interfaces appear more planar in samples irradiated at $300^{\circ} \mathrm{C}$ (Fig. 3) than those irradiated at liquid nitrogen temperature. The interface irradiated at liquid nitrogen temperature, moreover, also appears somewhat diffuse, although this is difficult to determine using HAADF-STEM. EDS line scans across the layers (Fig. 3(B)), however, support this premise. It should be noted that since EDS averages through the projected thickness, the effects of mixing and roughening are not easily distinguished. We note that the width of the mixed interface layer is considerably greater than the diffuse layers examined by $\mathrm{MD}$, however once the amorphous layer is formed it is reasonable to assume that the strength remains constant. This would explain why the interface strengths saturate by 5 dpa. A modified thermal spike model was previously employed to understand the temperature dependence of chemical mixing in the highly immiscible $\mathrm{Cu}-\mathrm{Nb}$ system.[11] Absent vacancy motion at liquid nitrogen temperature, chemical mixing occurs in thermal spikes and diffusional relaxation is inhibited. This enables thermal spikes in the vicinity of the interface to mix the two phases effectively.[16] Due to vacancy mediated diffusion at high temperatures, however, the large heat of mixing coupled with short-range diffusion can enable local phase separation. This interface may still structurally roughen, however, as longer range diffusion will not be active at $300^{\circ} \mathrm{C}$. Ion beam mixing studies suggest that $\mathrm{Cu}-\mathrm{Nb}$ interfaces amorphize during heavy ion irradiation below room temperature, but that they remain crystalline above $\approx 50-100{ }^{\circ} \mathrm{C}$.[12] Amorphization thus indeed appears to explain the convergence of interfacial shear strengths of samples at and below room temperature. The lack of amorphization at $300^{\circ} \mathrm{C}$, similarly, may explain why the interfaces irradiated at this temperature are weaker. 
In summary, damage accumulated at interfaces during fast heavy ion irradiation leads to an increase in the shear strength of $\mathrm{Cu}-\mathrm{Nb}$ interfaces. Irradiation damage, here primarily observed as interface roughening and chemical mixing, is greatest at low temperatures, where thermal vacancy migration is negligible. An increase in chemical diffuseness of the interface, as characterized by EDS line scans, correlates with an increase in interfacial shear strength. At all temperatures between -196 and $300{ }^{\circ} \mathrm{C}$ the measured interfacial shear strength saturates, within experimental error, at doses below 5 dpa. The results can be rationalized by past MD and ion beam mixing experiments that indicate that $\mathrm{Cu}-\mathrm{Nb}$ interfaces become amorphous during irradiation at room temperature and below..

This research was supported by the US DOE- BES under Grant DEFG02-05ER46217. It was carried out, in part, in the Frederick Seitz Materials Research Laboratory Central Facilities, University of Illinois.

\section{References}

[1] Zinkle SJ, Horsewell A, Singh BN, Sommer WF. J. Nucl. Mater. 1992;195:11.

[2] Demkowicz MJ, Hoagland RG, Hirth JP. Phys. Rev. Lett. 2008;100:136102/1.

[3] Enrique RA, Bellon P. Phys. Rev. Lett. 2000;84:2885.

[4] Chee SW, Stumphy B, Vo NQ, Averback RS, Bellon P. Acta Mater. 2010;58:4088.

[5] Zinkle SJ, Was GS. Acta Mater. 2013;61:735.

[6] Vo NQ, Averback RS, Ashkenazy Y, Bellon P, Wang J. Journal of Materials Research 2012;27:1621.

[7] Demkowicz MJ, Thilly L. Acta Mater. 2011;59:7744.

[8] Skirlo SA, Demkowicz MJ. Appl. Phys. Lett. 2013;103:171908/1.

[9] Wang J, Misra A. Current Opinion in Solid State and Materials Science 2011;15:20.

[10] Li N, Mara NA, Wang J, Dickerson P, Huang JY, Misra A. Scripta Materialia 2012;67:479.

[11] Averback RS, Peak D, Thompson LJ. Appl. Phys. A 1986;39:59.

[12] Aaen Andersen LU, Bøttiger J, Dyrbye K. Materials Science and Engineering: A 1989;115:123.

[13] Demkowicz MJ, Bhattacharyya D, Usov I, Wang YQ, Nastasi M, Misra A. Appl. Phys. Lett. 2010;97:161903/1.

[14] Ziegler JF, Ziegler MD, Biersack JP. Nucl. Instrum. Methods Phys. Res., Sect. B 2010;268:1818.

[15] Özerinç S, Averback RS, King WP. Journal of Nuclear Materials 2014;451:104.

[16] Zhang L, Demkowicz MJ. Acta Materialia 2014;76:135. 
Fig. 1: Time-lapse images of nanocompression test performed on an as-deposited $\mathrm{Cu}-\mathrm{Nb}$ nanolaminate presented along with the load-displacement curves for the sample.

Fig. 2: Interfacial shear strength plotted as a function of dose in units of dpa.

Fig. 3: HAADF-STEM images of $\mathrm{Cu}-\mathrm{Nb}$ multilayers as-deposited, and after $\mathrm{MeV}$ $\mathrm{Kr}^{+}$irradiation to $5 \mathrm{dpa}$ at $-196{ }^{\circ} \mathrm{C}$ and $300{ }^{\circ} \mathrm{C}$, along with associated EDS line scans across an individual $\mathrm{Cu}-\mathrm{Nb}$ interfaces. 

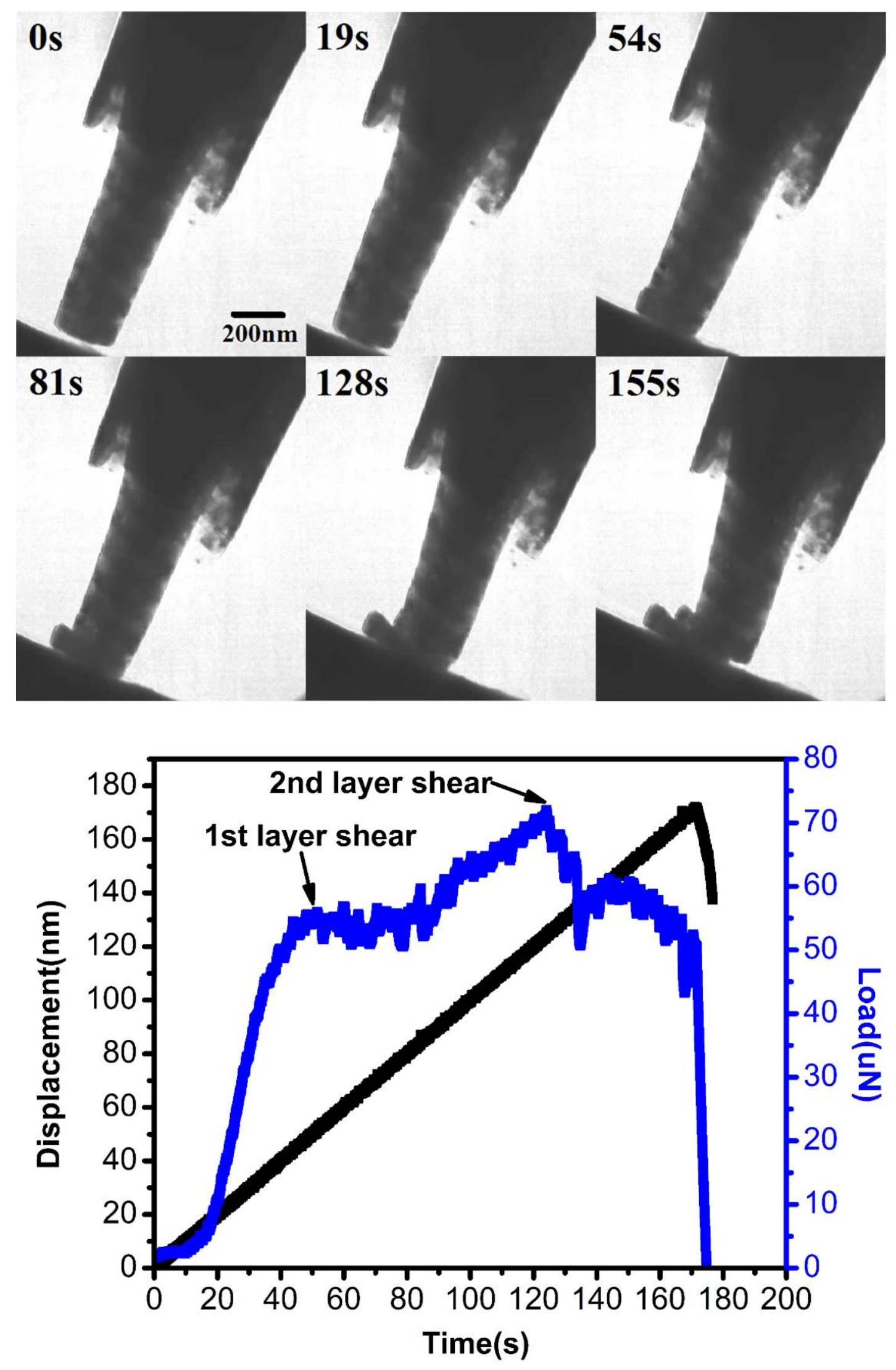


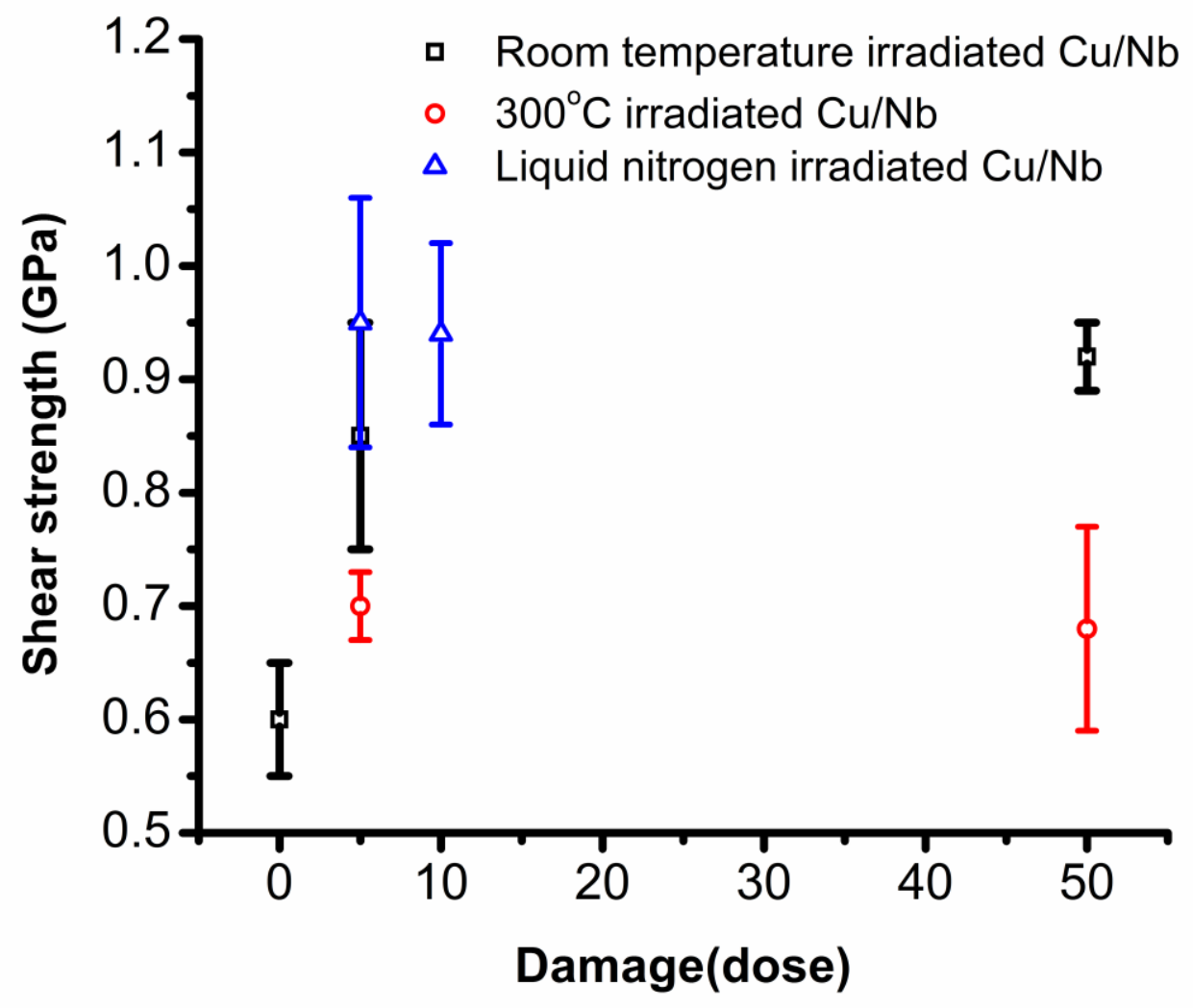



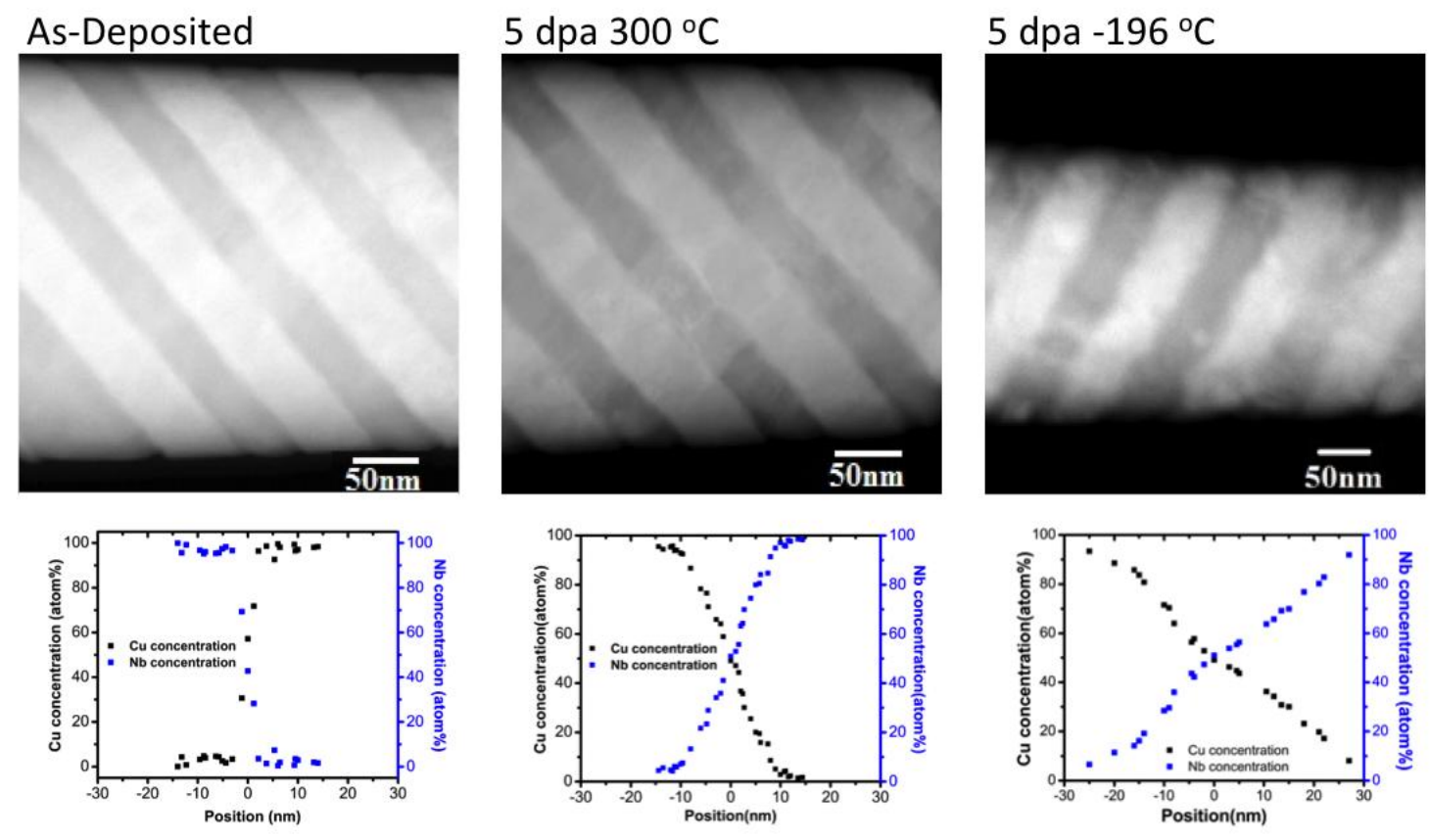